\title{
Aspectos Económicos de la Flexibilidad Temporal
}

Albert Recio Andreu *

RESUMEN:

Este trabajo analiza las razones económicas de la flexibilidad laboral en general $y$ de las diversas modalidades de flexibilidad temporal en especial. Se muestra como la misma obedece a las respuestas empresariales a diferentes aspectos del mercado de productos y a la büsqueda de rentabilidad. Y se muestra que el planteamiento obvia tanto las causas patológicos que inciden en las demandas de adaptación como los costes sociales que generan determinadas politicas de flexibilidad temporal, proponiéndose formas alternativas de juzgar las políticas.

Palabras Claves:

Flexibilidad laboral, flexibilidad temporal, costes sociales, desigualdades sociales, organización productiva

- Profesor titular de Economia Aplicada. Universitat Autónoma de Barcelona • albert.recio@uab.es 
ABSTRACT:

This paper analyses economic arguments favourable to labour flexibility and, specially, to different forms of time flexibility. It is oriented to show that their implementations are related to employers responses to different aspects of product markets and the search of profitability. And it is oriented to underline that economic reasons tend to forget, both, that there are some pathology in many causes of economic variability and buge social costs related with time flexibility, introducing some proposals in order to introduce a diverse political solutions.

KEY WORDS:

Labour flexibility, Time flexibility, Social costs, Social inequalities, Productive organisation

\section{INTRODUCCIÓN}

Toda actividad humana tiene una dimensión espacio-temporal. Pero las formas como nuestra actividad se relacionan con el tiempo y el espacio han cambiado poderosamente a lo largo de la historia a medida que nuevas tecnologías y cambios sociales han cambiado las condiciones de realización de distintas actividades. Como es bien conocido los inicios de la era capitalista inauguraron una nueva relación de la actividad humana con tiempo (y espacio) dando lugar a una nueva civilización.(Thompson, 1979) La generalización del uso del reloj y del trabajo en un espacio ajeno a la mayoría de individuos (la fábrica), caracteriza este cambio. Un cambio posible por la combinación de innovaciones técnicas e intereses sociales. La historia de la sociedad capitalista es, en parte, una historia de las transformaciones y conflictos en torno a los aspectos espacio-temporales de la organización social. En los últimos veinte años hemos asistido a una nueva oleada de cambios tecnológicos, sociales y organizativos que se han planteado en términos de flexibilidad. En este artículo tratamos de analizar los aspectos cruciales que afectan a este debate, especialmente a lo que se refiere a la flexibilidad temporal.

\section{LA CUESTIÓN DE LA FLEXIBILIDAD: UNA VISIÓN GENERAL}

Flexibilidad laboral es un término puesto en circulación a principios de los años ochenta.(Boyer, 1985; Bruno, 1989; O.C.D.E. 1986, 1987) Se planteaba como un elemento básico para superar los graves problemas de empleo que dominaron un periodo en el que el paro masivo y los cierres empresariales volvieron a entrar en el universo cotidiano de millones de personas en los países desarrollados. Se introducía un concepto que trataba a la vez de criticar el modelo laboral productivo que había conducido a la crisis de los setenta y proponer vías de salida en el campo laboral. 
En el planteamiento del propio concepto participaron diversas tradiciones culturales. En gran medida el planteamiento de la flexibilidad estaba asociado al resurgir de la escuela neoclásica-liberal que había permanecido en segundo término durante el período de hegemonía del pensamiento keynesiano (por más que el planteamiento dominante en el período 1950-75 no fue ni el keynesianismo ortodoxo ni el post-keynesianismo sino el pensamiento de la llamada síntesis neoclásica). Pero en el debate también tomaron parte activa muchos investigadores que se proclamaban de tradiciones institucionalistas o neomarxistas, como es el caso de Piore (Piore y Sabel, 1984; Piore, 1986) o Boyer (Boyer 1985). Aunque a menudo unos y otros se referían a cosas diferentes, acabaron construyendo una representación de la situación económica bastante común.(Recio, 1994)

La idea de flexibilidad es a la vez sencilla y compleja. En la vida cotidiana sugiere al menos dos cuestiones a) capacidad de adaptarse al cambio. Aunque esta adaptación no siempre se entienda como adaptación para mejorar (por ejemplo, flexibilidad es aprender una actividad nueva- como el uso del ordenadorpara acceder a una actividad que nos permitirá nuevas posibilidades de actuación) sino que muchas veces se trata de aceptar los mil y un avatares que nos da la vida (por ejemplo; alguien que ha tenido un accidente que le ha provocado una minusvalía debe ser capaz de adecuar su vida a su nueva situación) b) capacidad de ceder en nuestras pretensiones, aceptando las razones de los otros y renunciando a imponer nuestra voluntad (por empatía o por considerar que el coste de hacerlo es excesivo o éticamente indeseable). La idea es sencilla pero las formas de aplicarla son complejas. La respuesta flexible puede darse de muchas formas, cada una con consecuencias diferentes para los distintos sujetos implicados. Pero en todo caso en la vida cotidiana la idea de flexibilidad goza de buena valoración social: la mayoría de la gente suele considerar mal a las personas incapaces de adaptarse a las circunstancias o de ceder en sus exigencias, aunque sobre ello no siempre existe una única vara de medir: suelen estar mal vistas las personas egoístas, insensibles a las necesidades de los demás (inflexibles). Aunque puede ser bien valorado la persona que en su comportamiento cotidiano se muestra inflexible ante exigencias indeseables: Todo el mundo querría ser representada por políticos incorruptibles (que no fueran flexibles a las voces de sirena de los grupos de presión privados) y suelen ser admiradas las figuras que no se doblegan a las imposiciones de los sistemas autoritarios. Pero salvando casos especiales la flexibilidad tiene una connotación positiva. En buena medida porque la vida cotidiana de la gente común está dominada por una necesidad continua de adaptación a los mil y un vericuetos de la convivencia. Por esto el concepto de flexibilidad laboral acarreaba un notable elemento propagandístico que impedía un debate más analítico sobre su bondad. 
La idea de flexibilidad laboral desarrollada en la década de los ochenta incluía de hecho los dos elementos señalados anteriormente: adaptación y concesión. Adaptación en el sentido de cambiar las condiciones de trabajo en función de cambios en el mundo exterior. Un mundo exterior entendido como un mercado impersonal que "exige" o "impone" cambios a las empresas individuales y estas deben dar respuestas adaptativas a los mismos. Concesión porque se partía de la consideración que el principal factor que explicaba la incapacidad para adaptarse a estos cambios era debida a un exceso de protección de las condiciones de trabajo de las personas los cuales dificultaban la respuesta rápida a los mismos- Por esto una parte de la estrategia flexibilizadora consistía en demandar a los asalariados y sus representantes que aceptaran un recorte en sus derechos (o lo que es lo mismo que concedieran más derechos discrecionales a las empresas) para poder realizar esta adaptación que se consideraba ineludible. Se trataba de una verdadera concesión porque en la mayoría de los casos no había una compensación en otras cuestiones (por ejemplo salarial), sino que a menudo la única contrapartida que se ofrecía era el mantenimiento del empleo.

\section{Las demandas de adaptación}

La flexibilidad laboral se presenta tanto como una necesidad de adaptación a los cambios del mercado como una potencialidad para mejorar el bienestar social.

Como factores de necesidad destacan la recurrencia de "shocks" que alteran la estabilidad económica y la importancia del nuevo marco competitivo internacional. La primera cuestión indicaría que las empresas se mueven en un contexto de mayor inestabilidad económica que fuerza una continua adaptación de sus planes de producción. El argumento se sostiene siempre que se tome la "volatilidad" de los mercados como un dato inapelable y no como el resultado de una mala organización económica. Como ha señalado Stiglitz (2002) es posible que la inestabilidad reciente haya estado en parte producida por las políticas impulsadas por el F.M.I...Quizás lo sensato sería cambiar estas políticas. La segunda cuestión es la de la competencia internacional. Aunque hay motivos para pensar que el nuevo marco internacional exige ajustes más rápidos (adaptación a los clientes, fluctuacines de los tipos de cambio...), más que nada parecen estar exigiendo concesiones de derechos y salarios para abaratar precios y frenar deslocalizaciones.

Volatilidad y competitividad se consideran costes ineludibles de un proceso de especialización productiva a escala planetaria del que los economistas neoclásicos solo esperan buenas noticias. Minusvaloran los costes o incluso atribuyen los "shocks". a elementos externos al mercado (por ejemplo al Qaeda o los virus asiáticos). Olvidan que los mercados reales pueden ser regulados de formas muy diversas e ignoran que son las decisiones privadas de inversión las principales causantes de ines- 
tabilidad (Davidson, 1994). Una inestabilidad acentuada con la particular liberalización de los movimientos de capitales financieros. Se elude con ello las alternativas orientadas a reducir los impactos negativos de las incertidumbres financieras y la competencia destructiva (mejorando por ejemplo los derechos sociales)

La segunda línea justificativa de la flexibilidad tiene mayor calado. Se sugiere que una organización flexible será capaz de satisfacer un abanico más amplio de necesidades- en cuanto a la composición de los productos, en el tiempo en el que están disponibles, en su disposición territorial- y de ofrecer una mayor variedad de bienes y servicios la vida más amena y adaptada a los gustos personales. Las adaptaciones y concesiones que se exigen al mundo laboral flexible serian el coste necesario a pagar para obtener un mayor nivel de satisfacción de las necesidades humanas..

En este análisis se obvian cuestiones claves. La primera es la propia consideración antropológica que subyace en la idea de variabilidad y amplitud de las satisfacciones, en que medida las mismas son necesidades "autónomas" de las personas o resultado de compulsiones inducidas por la presión de la propaganda, la emulación o los procesos de diferenciación social (Scitovsky, 1976; Esteve 1991; Lavoie, 1992; Doyal y Gough, 1994). La segunda es la cuestión distributiva.. En una sociedad donde persisten enormes desigualdades en el acceso a la propiedad, en la distribución de los ingresos, en el entramado de relaciones sociales la diferenciación productiva no puede separarse de a quién favorece la misma. El mercado no es un proveedor universal de bienes y servicios sino un proveedor selectivo. Una mayor diferenciación productiva bien puede derivar en mayores desigualdades. En la medida en una parte de nuestras necesidades son satisfechas en marcos extramercantiles (la producción familiar-doméstica, las aportaciones del entorno social, el sector público) hay también que considerar el impacto de la flexibilidad laboral sobre el funcionamiento de estos espacios, un debate permanentemente eludido por los enfoques económicos dominantes.

Para algunos la irrupción de las nuevas "tecnologías de la información" (informática-telecomunicaciones- electrónica) la principal responsable del cambio, puesto que habrían posibilitado a la vez la producción flexible y la globalización. (Castells, 1999).

\section{Las formas de la flexibilidad}

La flexibilidad laboral y la adaptación al cambio puede conseguirse por vías diversas:

- ajustes de plantilla en función de las variaciones de la producción

- movilidad interna dentro de la empresa. Esta a su vez podía ser funcional (entre 
distintos puestos de trabajo) o geográfica (entre distintos centros de trabajo de la misma empresa)

- ajustes horarios en función de las necesidades de la producción, una amplia gama de posibilidades que analizaremos posteriormente

- variaciones salariales ligadas a los cambios en el ambiente económico, lo que puede concretarse de formas muy diversas: aumento de las partes variables del salario, pago de rentas en función de los beneficios empresariales etc.

- adaptación a los cambios tecno-productivos mediante un proceso de formación y cualificación permanente.

En la práctica la mayoría de políticas de flexibilidad se han concentrado en las cuatro primeras formas, mediante reformas legislativas, negociación colectiva o mera imposición empresarial. En este último caso especialmente a través de nuevas formas de organización como la subcontratación y la departamentalización (Harrison, 1997; Recio, 2001). Aunque las formas flexibles de fijación de salarios solo se han desarrollado parcialmente, las desigualdades salariales han crecido por múltiples vías: fragmentación de la negociación colectiva mediante la externalización, debilitamiento sindical, introducción de escalas salariales diversas, eliminación de pagos suplementarios por determinadas actividades etc. (Alós y Jodar, 1998). Y, como veremos, esta presión sobre los salarios incide también en la introducción de otras medidas de flexibilidad.

\section{FlexibILidad y tiempo de trabajo}

Todo proceso productivo tiene una dimensión temporal. En las sociedades capitalistas el control sobre la fijación de la jornada de trabajo ha constituido uno de los ejes sobre los que se ha pivotado el conflicto social. No es por tanto extraño que uno de los aspectos más importantes sobre los que se han movido las políticas flexibilizadoras haya sido el del control del tiempo de trabajo, en sus diversas acepciones

Como norma general el planteamiento de la flexibilidad en este terreno lo que pretende es aumentar la disponibilidad laboral de asalariados y asalariadas en manos de la empresa. Una disponibilidad que se traduce en cuestiones diferentes y que en acaba produciendo jornadas laborales más largas, más cortas y variables según los casos

Detrás de estas políticas no solo subyace una cuestión cuantitativa que podría explicarse en los viejos términos de la "plusvalía absoluta": cuanto mayor es la jornada laboral mayor la rentabilidad, sino también la creciente conciencia de que el tiempo no es necesariamente una magnitud homogénea en el que las horas de trabajo son perfectamente intercambiables, sino que no sólo cuenta el volumen total de horas, sino las horas concretas en las que se aplica la actividad. 
En las economias precapitalistas el tiempo no era homogeneor. En las actividatdes agricolas la actividad laboral debe aplicarse especialmente en momentos específicos, según el clima y los ciclos natumales de los vegetaless y los animales. En las tareas agricolas el trabajo en julio no era intercambiable por el de diciembre, por cuanto en un mes se necesitaban muchas manos para realizar una activiclad como la cosecha, que exige un importante volumen de cooperacion simple en pocos dias y en otro habia pocas tareas a realizar. El trabajo tradicional de los ganaderos, aún hoy, tiene un cierto pareciclo con el trabajo doméstico, puestor que los animales requieren cuidados especiales (limpieza, comida, orcleño...) en horas concretas del día, lo que provoca a la vez una jormada laboral discontinua y una dedicación perpmanente. Los cambios técnicos ya comentados permitieron a las coonomias inclustriales sortear en buena medida esta dependencia de los "tiempos naturales" y organizar la actividad productiva mercantil de forma mis regular. Auncpue no lo han hecho de forma absoluta, puesto que no soblo han persistido formas de empleo ligadas a pautas temporales especificas (p.ej. estacionales) o simplemente muchas de estas actividades se han mantenido fuera de la cisfera mercantil, como tralsajos domestico-familiares.

Durante años la fijación de la jornada laboral posiblemente ha dépendiclo más de cuestiones derivadas de las regulaciones puiblicas y la negociacion colectiva, que de cualquier otra consideración. La empresa fordista sc plantealaa a si misma como un proceso en continua producción. con una planificalción de la actividad productiva y un ajuste por medio de las politicas de stocks, que se extendia a las recles comerciales y a los suministradores. Fvidentemente ello no siempre era así. Lats industrias más relacionadas con los ciclos naturalés, particularmente la inclustria alimentaria (en algunos paises la construcción y su industria auxiliar) siempre ha experimentado una peculiar estacionalidad. Pero en los últimos años se ha producido un cambio bastante radical en los tiempos de trabajo mercantil, en gran medicla asociado a la expansión de las actividades mercantiles a los servicios, que ha puesto en evidencia la heterogeneidad del tiempo y la necesiclad de organizar, en aras a la rentabilidad, la actividad empresarial atendiendo a especificas pautas temporaless. Una reflexión que también se ha extendido a la industria a partir de la crisis de Ios setenta cuando la experiencia de uná acumulación cle existencia clio lugar a pérclidas y empezaron a estar disponibles nuevas tecnologías de comunicación. La ordenación de la jornada laboral volvió a ser un campo central en las políticas de organización del trabajo (Bosch, 1995). Este cambio en la organización del tiempo de trabajo mercantil adopta cuadro formas básicas que pasamos a analizar:

\section{La jornada expandida}

Una de las vías de los nuevos modelos de gestión lo constituye la búsqueda de la expansión de las horas de actividad de la empresa. En el limite el funcionamien- 
to de la misma las 24 horas de día los 7 días a la semana. Existen dos grandes razones que apoyan esta pretensión:

a) Una política de abāratamiento de los costes de capital. El capital fijo constituye desde siempre la parte mayor de la inversión capitalista y su componente más complejo. Las inversiones requieren recursos financieros que a menudo exceden de las propias capacidades de la empresa y exigen una buena conexión en el mercado financiero. Estas inversiones se encarnan en bienes específicos, orientados a la producción de bienes particulares que se realizan con combinaciones particulares de inputs Ello supone que la inversión está siempre amenazada por la obsolescencia que supone la pérdida de atractivo del producto en el mercado o la entrada de nuevos competidores que pueden producir más barato mediante nuevas tecnologías que suponen diferentes combinaciones de inputs productivos (o situadas en territorios donde, por razones diversas, los costes de estos inputs son inferiores). Cuando surge un problema de obsolescencia la empresa esta abocada a liquidar con pérdidas la inversión puesto que en la mayoría de los casos la especificidad de sus bienes de equipo le impiden utilizarlo para otros fines o venderlo en un mercado de segunda mano, De aquí que la mejor política empresarial sea acelerar la amortización de los bienes de equipo como forma de minimizar los riesgos de la inversión. Y este objetivo se consigue cuanto más intensivo es el uso del mismo en un mismo espacio de tiempo (o sea cuanto mayor sea la producción que se obtiene del mismo por unidad de tiempo).

La utilización intensiva de un equipo de capital reduce, además los costes medios de producción. Habitualmente los equipos productivos requieren el uso de cantidades fijas por unidad de producto de otros inputs. A corto plazo puede considerarse que los costes de estos últimos están dados y de ello se deducen costes variables medios constantes. Pero si se permite una mayor utilización de este mismo equipo en un período dado, los costes fijos medios (y los totales) decrecerán. En algunos casos ello puede conseguirse con una aceleración del ritmo de actividad de equipos y trabajadores, con el consiguiente aumento de la intensidad del trabajo. Pero ello tiene límites. Y la forma más simple es aumentar el tiempo operativo del equipo hasta su plena utilización. No siempre es posible, las tareas de mantenimiento obligan a paradas obligatorias, pero estas tareas son más fáciles de integrar cuando mayor es el número de horas que la empresa tiene disponibles. El cambio tecnológico acelerado (real o simplemente imaginado) y la globalización, que aumenta el temor a la competencia y la incertidumbre de la vida económica, aumentan la necesidad de amortizar velozmente el capital físico mediante una utilización intensiva del mismo (Bosch y Lendorff, 1997; Muñoz del Bustillo et al. 2003)

La segunda razón para el alargamiento de la jornada la plantean las actividades basadas en la disponibilidad ante demandas que se producen de forma más o menos aleatoria. El comercio o los servicios de bomberos o de urgencias operan bajo 
este modelo. La actividad laboral se activa cuando aumenta el flujo de demandas, pero como este es imprevisible, el "productor" debe estar en situación de espera. Algunas de estas actividades responden a necesidades tan básicas y urgentes que está justificada la existencia de un servicio disponible de forma permanente, como es el caso de los diversos servicios dedicados a emergencias. En otros la jornada ha sido tradicionalmente limitada por razones institucionales, aunque siempre han existido tendencias a ampliarla. Por esta razón tradicionalmente muchas de estas actividades han sido cubiertas por trabajadores autónomos dispuestos a dedicar gran parte de su tiempo a obtener ingreso, en unos casos por codicia y en otros por necesidad (como es el caso de muchos vendedores del tercer mundo) (Muñoz del Bustillo et al. 2003).

La extensión de este tipo de actividades al mundo asalariado es en parte un subproducto de la entrada de la empresa capitalista en campos tradicionalmente dominados por la empresa familiar. En algunos casos, como es la campaña por la libertad de horarios comerciales, este es el campo donde se ventila el crecimiento del gran capital privado a costa de la pequeña empresa familiar: el comercio de gran superficie, habitualmente situado "lejos" de los lugares de residencia, requiere tiempo suficiente para que las gentes se desplacen, algo que suele ser posible los fines de semana. El crecimiento también se ha producido por las propias presiones competitivas nacidas de fuentes diversas: la desregulación de servicios públicos, las políticas de subcontratación de servicios de las grandes empresas, las políticas de captación de clientes con perfiles particulares...

El resultado es una enorme presión en la organización de servicios de 24 horas o, como mínimo, de una gran franja horaria que acaba extendiéndose a una enorme variedad de servicios (Bettio, Rubery, Smith, 1996)

Si estas son las fuentes básicas que provocan el alargamiento de las jornadas operativas de muchas empresas, no pueden perderse de vista la existencia de otros factores menos relacionados con la textura particular de cada actividad. La presión por ampliar la jornada laboral por la realización de horas extras es una constante de la empresa capitalista y una opción habitualmente preferible a la ampliación de plantilla. Desde el punto de vista empresarial existen diversas razones a favor de la opción horas-extra. Primero, la existencia de costes de contratación - selección de personal, formación en la entrada, indemnización por despido y, en algunos casos el coste de algún tipo de conflicto. Estos costes son nulos en el caso de la plantilla propia. Ello sin contar, en algunos casos, los "costes" que para las empresas puede tener el hecho de superar un determinado nivel de empleo (por ejemplo en materia de representación sindical). Segundo, menor dilación temporal en los ajustes. Las horas extra se pueden aumentar o reducir a discreción siempre que exista un ambiente propicio, la contratación de nuevo personal puede tener efectos más lentos, por cuanto habrá como mínimo un tiempo de búsqueda y adaptación de los 
nuevos empleados y el despido en algunos casos supone en muchos casos un proceso de cierta duración. Tercero, en algunos casos la política de horas extra puede formar parte de la política de costes salariales de la empresa. Ello es especialmente importante cuando las empresas pagan salarios bajos y presentan las horas extra como una forma de "complementar" los ingresos. Por esto la realización de horas extra constituye una práctica habitual de las empresas, solo frenada por regulaciones externas.

En los modelos neoclásicos convencionales el mecanismo que induce a las personas a aceptar más horas extras es la comparación entre el precio relativo del ocio (el salario dejado de ganar) y el consumo que puede obtenerse con más horas de trabajo.(McConell, Brue, 1996). Ello explica que el plus salarial que a menudo tienen las horas extra se considere un acicate al alargamiento de la jornada laboral. Este análisis omite otras cuestiones que pueden ayudar a entender por que en países como España la jornada laboral efectiva esté creciendo a pesar de reducirse en la negociación colectiva.

En primer lugar esta la cuestión de cual es el mecanismo que hace decidir a la gente si quiere trabajar más o menos. Para los economistas neoclásicos este es funclamentalmente el coste relativo de ocio-consumo (la inversa del ocio, la desutilidad del trabajo) evaluado en términos individuales. Este análisis suele minusvalorar la importancia de los efectos-renta y el papel que juegan los estándares de vida (y con ellos el nivel de gasto absoluto) a la hora de condicionar las opciones laborales de la gente. El punto de partida es que en cada sociedad moderna existe un nivel de gasto difícil de reducir (al igual que existe un nivel de trabajo doméstico clifícil también de eliminar). Y el objetivo primario de la actividad laboral de las familias es alcanzar este mínimo de ingresos que les permiten acceder a un nivel cle vida digno. Hay sin duda gastos ajustables (por ejemplo el gasto alimentario según el tipo de dieta escogida, o el de vestido), otros lo son menos (particularmente para personas jóvenes el coste de la vivienda y el pago de una hipoteca parece en nuestro país difícilmente eludible) y globalmente hay un mínimo de ingresos que según el tamaño de la unidad familiar no pueden eludirse. Dado el salario-hora imperante las personas ajustaran (si las condiciones de empleo lo permiten) trabajar todas las horas necesarias hasta alcanzar este nivel de ingresos, algo que harán siempre que el número de horas sea igual o menor al disponible para trabajar. De esta forma una caída de los salarios medios (o simplemente de los salarios de determinados colectivos de trabajadores), o un aumento de los gastos básicos de supervivencia, puede provocar una mayor aceptación de las horas-extra a pesar de que su precio no aumente o incluso disminuya. Ello abre la posibilidad de que las empresas usen esta situación para elaborar políticas que aumenten la jornada laboral bien ofreciéndolas como una posibilidad de complementar ingresos, bien presentando sus condiciones de trabajo como un "paquete" en el que se incluye 
una jornada laboral más larga y una remuneración total (como parece haber sido habitual en el sector turístico). En ambos casos la aceptación de esta jornada expandida aparecerá como voluntaria si se olvida el papel que juega un salario hora muy bajo.(George, 2000; Prasch, 2000). En segundo lugar no puede descartarse que la aceptación de jornadas más largas sea el mero resultado de algún tipo de coacción empresarial. La extensión de los contratos temporales en los que las empresas tienen el poder de renovar cada cierto tiempo (o de ofrecer tácitamente el paso a fijo) o, en otro campo la contratación de extranjeros pendientes de la renovacíón de permisos o simplemente ilegales, constituyen medios poderosos que pueden forzar el alargamiento de la jornada. Algunos trabajadores indican incluso mecanismos más sutiles, especialmente en actividades de servicios en las que junta a la empresa actúa un tercer elemento, el "cliente". La jornada puede prolongarse porque el empleado se siente apremiado (directamente o simplemente por propio sentido de la responsabilidad) a cubrir el servicio. Ello es fácil que ocurra cuando la empresa contrata una plantilla subóptima que automáticamente genera una tensión y una carga de trabajo extra para los empleados. En tercer lugar, existe un poderoso mecanismo para forzar jornadas laborales más largas (y que explica, por ejemplo su existencia en sectores donde predomina el empleo estable y los salarios son relativamente altos, como es el caso del sector financiero). Se trata del mecanismo de promoción y carrera profesional. En la medida que las empresas tienen el poder de promocionar a unos y otros asalariados, que estos perciben su vida profesional como una "carrera" hacia la cúspide (algo a lo que ayuda la existencia de una estructura salarial muy fragmentada y jerarquizada) es fácil (como mostró Akerlof (1986) en su modelo de la "carrera de ratas") obtener horas de trabajo adicionales a coste cero, realizadas en la confianza de que en el futuro ello se traducirá en un ascenso o plus salaria. En la medida que un sistema de este tipo se generaliza incluso personas con poco afán competitivo pueden verse presionadas por el ambiente a aceptar esta prolongación de la jornada laboral. No puede perderse de vista la relación de estas estrategias de promoción-prolongación de jornada con la desigual situación de hombres y mujeres en los segmentos altos del mercado laboral, y a la vez la presión que se produce en estos estratos sociales sobre las tareas y actividades de la vida doméstico-familiar.

La voluntad de las empresas de alargar el tiempo de operatividad, genera por sí misma la necesidad de obtener mayor disponibilidad a la ampliación de jornada. Aunque este aumento del tiempo de actividad puede en teoría cubrirse con turnos de trabajo bien definidos, cuanto mayor es el tiempo operativo mayores son las situaciones en los que la empresa pueda necesitar de un alargamiento de jornada: como es el caso de la cobertura de bajas o puntas de actividad imprevistas, la acumulación de faena en horas específicas etc. En este sentido la regulación de horarios puede estar influyendo sobre la demanda patronal de horas-extra. 
Por otra parte los diferentes aspectos de las políticas desreguladoras, con el aumento de las diferencias salariales, (o, como mínimo la persistente moderación de los sectores con salarios más bajos), el debilitamiento del poder sindical (por medidas directas o por efecto de los cambios impuestos por el cambio en el modelo de contratación laboral y la extensión de las subcontratas), el machacón discurso ideológico sobre las bondades de la flexibilidad etc. acaban por crear las precondiciones sobre las que es posible generar un alargamiento de la jornada laboral en muchas áreas del mundo productivo.

\section{La jornada recortada}

Paralelamente a las presiones por ampliar el espacio horario de la actividad laboral se ha producido la aparición en gran escala, incluso en España donde el fenómeno es más limitado, del empleo a tiempo parcial. Es posible que pàra algunas actividades y algunos países la novedad sea menor y se trate simplemente de un efecto estadístico provocado por los intentos de medir cualquier actividad remunerada en un período donde la actividad pública ha estado obsesionada con encontrar el crecimiento de la tasa de actividad, la emersión de la economía sumergida y la reducción estadística del desempleo. Tal es el caso, para España, de las tareas domésticas retribuidas, Una actividad con larga tradición y que constituye la principal bolsa de empleo femenino a tiempo parcial. Pero más allá de estos fenómenos es innegable que el empleo a tiempo parcial se ha tendido a extender hacia otros campos de actividad.(Fina, 1997; O’Reilly y Fagan, 1998)

Se pueden postular dos razones para el empleo a tiempo parcial. Las originadas por la demanda, por las características de determinadas actividades y las originadas por la oferta, las características o necesidades de los asalariados.

Las razones de la demanda tienen que ver con la heterogeneidad del tiempo de vida real y la imposibilidad de organizar muchas actividades como una secuencia ininterrumpida. En muchas actividades se producen espacios donde la actividad se concentra y se requiere un aumento de la cantidad de fuerza de trabajo requerida. Algo que como ya se ha comentando ha sido característico. de las actividades estacionales agrícolas y que también se encuentra en muchas actividades de servicio. Ello es particularmente importante en las que se han calificado como actividades de espera, donde suele ser habitual que los demandantes acudan con mayor intensidad en determinados momentos del tiempo. En el caso que la oscilación sea estacional la respuesta empresarial será demandar empleos temporales, pero en el caso de que la acumulación de tareas ocurra en espacios temporales de menor dimensión (aunque sean más recurrentes) la respuesta será la voluntad de contratar personas por el tiempo limitado en el que tiene lugar esta demanda. 
Esta temporalidad especial suele tomar dos formas diversas. Actividades concentradas en unas pocas horas al día. Tal es el caso los restaurantes, especializados en clientelas específicas, por ejemplo los de comidas de mediodía en áreas de fuerte concentración laboral. $\mathrm{Y}$ actividades concentradas en un número reducido de días a la semana. Este es por ejemplo el caso de los restaurantes y otras actividades de ocio que concentran su demanda los fines de semana.

En la proliferación de estas pautas juegan muchos factores. El principal es la creciente mercantilización de las actividades de servicios. Mercantilización que entraña además especialización. Cuando muchas de las actividades de servicios se cubren por trabajo doméstico, este se caracteriza (en el caso de una persona dedicada enteramente al mismo) en una suma de pequeñas actividades que se van realizando a lo largo del día. Cuando estas actividades son desarrolladas por el mercado, la lógica es la especialización. En algunos casos ello no entraña un perfil temporal específico (por ejemplo una empresa de lavandería puede organizar su actividad de forma continua como cualquier empresa industrial), pero si ocurre con otras que obedecen a pautas horarias basadas en necesidades condicionadas por la naturaleza o las convenciones sociales, como es el caso ya comentado de la comida y de la mayoría de actividades de cuidados personales.. En esta acumulación de tareas en momentos del tiempo se deriva de que la gran mayoría de servicios requieren de la presencia personal del "cliente" y por tanto este sólo acudirá a dichos servicios cuando tenga tiempo para ello. En la medida en que una gran parte de actividades están organizadas en pautas temporales rígidas (no sólo las laborales, también las educativas) y que estas se combinan con las pautas de la vida personal (tiempos de descanso nocturno, etc.) el resto de actividades se concentran en un horario residual donde se producen "aglomeraciones". Posiblemente en algunos campos este efecto "concentración" este reforzado por las pautas de desarrollo espacial, como es el caso de la ubicación de actividades comerciales y de ocio en espacios alejados de los residenciales. Su lejanía no sólo genera una clara dependencia del automóvil privado, también ayudan a que la actividad se concentre en los fines de semana. En todos estos campos existen grandes posibilidades de la aparición de este factor aglomeración y de la consiguiente demanda de asalariados a tiempo parcial.

Puede también postularse que el crecimiento del empleo a tiempo parcial se explica por razones de oferta de fuerza de trabajo, particularmente como reflejo del aumento de mujeres que acuden al mercado laboral. De hecho está es la motivación de algunas políticas públicas. Si hay mujeres que aún tienen como eje de su actividad el trabajo doméstico pero que están dispuestas a complementar los ingresos familiares con un porcentaje de trabajo mercantil las empresas tienen la oportunidad de obtener una fuerza de trabajo adicional a un coste posiblemente menor, dada la particular regulación del empleo a tiempo parcial en muchos países. 
Hay sin embargo algunas evidencias que muestran que este motivo sólo puede operar de forma secundaria. En primer lugar, el empleo a tiempo parcial se ha desarrollado en un período de desempleo masivo donde en muchos casos las empresas no han tenido graves dificultades para encontrar trabajadores y por tanto no han sentido la necesidad de cambiar sus pautas de contratación laboral. En segundo lugar, la contratación de personas con diferentes pautas horarias aumenta los costes de coordinación y resulta poco eficaz allí donde el sistema productivo requiere un elevado grado de cooperación (o simplemente está organizado de forma rígida como en las cadenas de montaje). Sólo en actividades donde el trabajo se realiza en un plano meramente individual y resulta fácil de controlar tiene interés "ofrecer" diferentes pautas horarias. Las empresas tendrán en cambio interés en asumir los costes de contratación cuando a cambio resuelvan los problemas de aglomeración descritos anteriormente. En tercer lugar, los horarios más interesantes para las personas dedicadas prioritariamente al trabajo doméstico no son necesariamente compatibles con los horarios en los que las empresas están interesadas en contratar una fuerza de trabajo adicional y por ello, difícilmente pueden ofrecerse como alternativas. Ello no quiere decir que las empresas no puedan organizar su sistema productivo de forma que tengan acceso a una mano de obra, habitualmente femenina, que trabaja a tiempo parcial, pero ello requiere un proceso de diseño organizativo que sólo se da en circunstancias muy particulares del mercado laboral y en plantas de nuevo diseño (tales parecen ser las condiciones que se dieron en la planta de ferralla de Prefabricats Pujol, donde se creo un sistema completo de producción en turnos de tiempo parcial en una zona caracterizada por un relativamente bajo nivel de paro). En cuarto lugar la evidencia disponible muestra que en muchos campos, por ejemplo en el trabajo administrativo, el sector público ha jugado un importante papel en el desarrollo del empleo a tiempo parcial, lo que indica que su crecimiento se ha debido más a la lógica de las políticas públicas de algunos países que a las demandas de las empresas o el "mercado".

Hay que constatar por último que el empleo a tiempo parcial pueda, en algunos contextos, desarrollarse como resultado de condiciones particulares. Especialmente debido a la existencia, en muchos países, de regímenes laborales diferentes para el empleo a tiempo completo y a tiempo parcial. En determinados contextos ello puede dar la posibilidad a las empresas de utilizar estos contratos de forma "oportunista”, asegurándose con ellos una reducción de costes de la seguridad social y pagando con "dinero negro" el resto de la jornada laboral.

Aunque no puede perderse de vista, que en un contexto liberalizador la busca de soluciones específicas a problemas concretos se combina con todo tipo de operaciones orientadas a eludir el pago de impuestos y contribuciones fiscales, hay que considerar que es el cambio de modelo productivo la fuerza más sólida que condu: ce a la creación de empleos de horario reducido. 


\section{La jornada variable}

Otro de los cambios impuestos por los modelos flexibles lo constituye la introducción de "jornadas flexibles", de perfiles horarios que cambian en función de variaciones en la vida de la empresa. Un mismo volumen de horas de trabajo contratadas puede adoptar formas diversas. Esta variación adopta a su vez formas distintas

En primer lugar los turnos, que constituyen una forma tradicional de organización del trabajo allí donde las empresas trabajan en un ciclo continuo o semicontinuo. Los turnos han sido tradicionales en sectores específicos que, por diversas razones, operan a todas horas, como es el caso de los hospitales o los servicios de bomberos. También en empresas industriales, particularmente en industrias de proceso continuo, que tenían elevadísimos costes de arrancada, lo que hacía preferible mantener continuamente abierta la producción. Pero en la medida que en un gran número de sectores se adopta una política de ampliación del horario buscando una utilización más intensiva de la capacidad productiva o la ampliación del horario de servicio al público, es evidente que el consiguiente aumento de horas de trabajo acaba derivando en el aumento de los turnos. (Sloane, 1978)

Desde el punto de vista de la variabilidad del horario de trabajo, los turnos pueden ser estables, pero en muchos sectores se acaban adoptando turnos rotatorios con el fin de dar satisfacción a las demandas de muchos trabajadores que no desean verse condicionados a un permanente turno indeseable y consideran más aceptable la rotación. De hecho la propia ampliación del tiempo de operación de las plantas y establecimientos puede forzar esta variabilidad de la jornada laboral en la medida que la transferencia de trabajadores entre turnos puede permitir cubrir incidencias de la producción y la vida laboral. En todo caso cabe esperar que salvo sectores muy concretos esta es una forma menor de variabilidad.

La segunda fórmula, mucho más innovadora es la introducción de una jornada laboral variable (número de horas trabajadas a diario). En unos casos mediante la introducción de una jornada de duración variable a lo largo de un determinado número de días, en otros mediante un número de potenciales días adicionales de trabajo (por ejemplo un número de sábados al mes) compensables con dinero o con días de fiesta adicionales en otra época del año. En otros introduciendo la posibilidad de alterar la estructura de festivos, particularmente la realización de vacaciones. Esta variedad de fórmulas tiene en común que es la empresa la que decide, habitualmente dentro de los márgenes acordados, cuando se aplica la norma de flexibilidad, y por tanto cuando se altera el horario o se introduce un día adicional. Una variante particular de esta medida, al menos en España, lo consideran los días de regulación de empleo cuando la empresa considera excesivo el nivel de existencias o quiere reducir el volumen de producción, una política bastante habitual entre los constructores de coches. En este caso la empresa transfiere total o parcial- 
mente el coste salarial (totalmente cuando los trabajadores sólo cobran el seguro de desempleo- perdiendo la diferencia entre el salario que cobran en la empresa y la pensión de desempleo; parcialmente cuando la empresa asume esta diferencia) (Sayer, Walker, 1992; Moody, 1997)

En todas estas medidas de ajuste las empresas tratan fundamentalmente de reducir el impacto de los costes de almacenaje derivados de la estacionalidad de la producción y de la incertidumbre en la salida de productos. Unas políticas que se ven potenciadas en el modelo de producción flexible por varias razones. En primer lugar los nuevos modelos de producción "justo a tiempo" pretenden, en el límite, eliminar existencias (el modelo busca trabajar a pedido), ello supone que la actividad se realiza siempre en una situación de estrés del sistema productivo. Cuando la demanda es alta la empresa se ve obligada a forzar su actividad productiva lo que, dada una tecnología instalada, solo es posible mediante un aumento de las horas de ocupación. En los momentos de baja no tiene sentido seguir produciendo para acumular existencias y la única alternativa consiste en reducir o parar el nivel de actividad. El objetivo de las existencias cero es por una parte eludir los costes de almacenaje (equipo e instalaciones, costes financieros del capital circulante...) y por otra evitar la acumulación de productos que pueden tener una mala salida en el mercado. Hay que anotar también que esta situación de estrés (trabajar al tope cuando hay demanda, parar completa o parcialmente cuando esta frena) se acentúa por los problemas que puede tener la red de suministradores externos, si estos fallan en el suministro el flujo productivo se puede detener independientemente de que exista o no demanda. En segundo lugar la mayor variedad de productos aumenta la posibilidad de cambios en la demanda más rápidos e impredecibles, lo que aboca a las empresas a buscar políticas que les permitan responder con menor coste a estos cambios. Por último la constatación de las estacionalidades de muchas actividades combinado con la búsqueda de modelos de producción sin stocks refuerza aún más esta orientación, por cuanto los productores trataran de adaptar su ciclo productivo a la temporada comercial, no sólo para eludir las existencias, sino también para responder más velozmente a las maniobras de la competencia. Aunque se trata de ajustes menos automáticos que lo previsto aumenta el número de empresas que tratan de organizar la producción en esta dirección.

Existen variantes más radicales de este modelo. Especialmente en el sector servicios donde el problema de existencias no es tan importante pero donde abundan las variaciones tanto de la demanda (necesidades específicas de los clientes, días especiales...) como por la gestión laboral (bajas imprevistas del personal, alta rotación en los sectores de muy bajos salarios). En estos cambios el ajuste horario puede ser permanente y los períodos de comunicación de los cambios por parte de las empresas pueden ser muy cortos. En muchos casos la existencia de contratos a tiempo parcial facilita este tipo de ajuste porque la empresa cuenta con la ventaja de poder 
combinar de formas más sutiles las jornadas laborales de los trabajadores. Para algunas empresas cuenta, además, la ventaja de dificultar el control de la jornada efectiva por parte de los propios empleados (European Comission, 1998)

Aunque las dos primeras fórmulas suelen estar asociadas a una negociación, no cabe duda que el resultado de la misma no sólo ha aumentado el nivel de discrecionalidad empresarial, sino que además ha reducido el grado de control individual de las condiciones de trabajo. $Y$ no puede incluso perderse e vista que en muchos casos la variabilidad de horarios se convierta en una fórmula que permita a las empresas dislocar la organización de los trabajadores (dificulta su comunicación, la creación de una cultura colectiva) y reducir el control riguroso de la jornada laboral.

\section{La jornada "anormal": noches y dias de fiesta}

Existe un último efecto, más que una práctica particular es el resultado en parte lógico de las políticas que acabamos de describir. Se trata de un aumento de las personas que tienen horarios de trabajo "anormales" desde el punto de vista de la forma como las personas perciben la vida cotidiana y están organizadas muchas actividades.

Cabe señalar que esta es en parte una cuestión discutible. En sociedades tradicionales ya hemos indicado al principio que no existía una jornada de trabajo regular, sino que esta variaba a lo largo del tiempo en función de los ciclos naturales pero en todo caso se trataba de ciclos relativamente previsibles y más o menos regulares. Existían además una enorme cantidad de festivos en los que las prescripciones religiosas y las costumbres reducían la disposición a trabajar (aunque todas estas regulaciones no atendían al trabajo doméstico-reproductivo para el cual la única limitación la han impuesto los límites del standard de vida de cada momento). Parece evidente que la irrupción del capitalismo impuso una expansión del tiempo de trabajo hasta límites insospechados. Expansión que en las fases iniciales de la industrialización afectó a hombres, mujeres y niños. Para los antiguos asalariados su vida era sólo una vida de trabajo (algo que también afectaba a esta categoría de "asalariados-siervos" que eran los criados, uno de los grupos laborales más numeroso). La paulatina lucha sindical y política tendió a acotar no sólo la jornada laboral máxima sino su configuración con la introducción de innovaciones como la semana inglesa (día y medio de fiesta) o las vacaciones regulares. Ello no suponía la inexistencia de franjas con horarios diferentes, sino en todo caso que estos horarios fueran considerados "anormales" y reservados a unas actividades especiales. O que exigían un nivel de prestaciones diferentes por parte de las empresas en el caso que quisieran movilizar mano de obra fuera de la jornada regular.

La importancia de las políticas de flexibilidad es que quiebran la diferencia entre jornada regular y jornada atípica y eliminan gran parte del garantismo que ge- 
nera esta diferencia. Con ello no sólo se permite un uso creciente de las jornadas en festivos, nocturnos cómo fórmula laboral normal, sino que se abarata su coste al dejar de constituir piezas excepcionales de la vida social.

Muchas de las políticas empresariales descritas anteriormente generan un aumento del trabajo nocturno y en festivos. Un resultado esperable de una lógica productiva que pretende la maximización del uso del capital productivo y la ampliación del tiempo de oferta de los servicios. Un modelo que exige una disponibilidad que no sólo se limita a la presencia física en el lugar de trabajo sino que mediante el recurso a los nuevos medios de comunicación extiende la movilización potencial de la fuerza de trabajo hasta el espacio de vida privado que es movilizado ante situaciones de "emergencia" (en muchos casos más probables cuanto mayor es la oferta de servicios que plantea la empresa).

\section{EVALUAR LA FLEXIBLIDAD TEMPORAL}

Desde las posiciones económicas dominantes las políticas de flexibilidad temporal son plenamente eficientes en diversos espacios:

- mejoran la rentabilidad empresarial al permitir una amortización más rápida del capital invertido y reducir otros costes (gastos de almacenaje, tarifas eléctricas reducidas por la noche, etc.)

- mejoran el bienestar social al aumentar la oferta de servicios (en todas aquellas actividades en las que permite ampliar el horario activo), y adaptarlos a las necesidades de cada cliente (en su aspecto horario, en su personalización)

- mejoran las oportunidades de empleo para cubrir mayores franjas horarias

- pueden facilitar la reducción de la jornada laboral, en la medida que las ganancias en productividad, costes y demanda pueden absorber los costes que genera la reducción de la jornada. En algunos casos incluso se plantea la reducción de jornada como el mecanismo que permite a la empresa un diseño más flexible de su actividad que deriva en creación adicional de empleo.

Los mismos sindicatos participan de estos puntos de vista, en particular del último, aunque, en conjunto, sus políticas oscilan entre la propuesta activa de flexibilizar y reducir la jornada laboral y el mero intento de tratar de contrarrestar los peores efectos de su implantación. Pero a mi modo de ver el debate está incompleto si junto a las ventajas de la flexibilidad temporal no se evalúan sus potenciales efectos negativos:

\subsection{Costes ENCUBiertos}

Las políticas de flexibilidad han significado un cambio en los "derechos" de distintos sujetos sociales. $\mathrm{Y}$ han abaratado el precio al que los empresarios acceden 
a determinados usos de la fuerza de trabajo. Un abaratamiento que en parte puede explicarse por que no se contabilizan sus "externalidades"

Hay varias cuestiones relevantes al respecto, Especialmente los problemas de salud, del que existen evidencias en el caso del trabajo nocturno o los turnos. Los costes se transfieren a la población afectada y a los servicios públicos de salud. Pero también otros posibles efectos como el impacto sobre los modos de transporte u otros servicios públicos.

\subsection{IMPACTOS SOBRE LA VIDA COTIDLANA Y El TRABAJO FAMILIAR DOMÉSTICO}

El supuesto sobre el que se basa la bondad de la flexibilidad laboral es que el uso del tiempo libre es estrictamente individual y depende exclusivamente de la cantidad de tiempo disponible. Se supone que las personas son indiferentes al día de la semana que tienen libre, la disponibilidad de horarios diarios etc. Un análisis de lo que se realiza "al otro lado del empleo asalariado" muestra lo inadecuado de este supuesto. La mayoría de tareas domésticas tienen delimitaciones temporales bastante estrictas. La casi totalidad de actividades que dan sentido a la vida de la gente: cuidar de los demás, relacionarse, participar en la vida social y política, participar en actividades de ocio... se realiza en momentos específicos del tiempo y en contacto directo con otras personas. La alteración de los horarios laborales distorsiona la vida personal y crea graves problemas a nivel personal y social. (Aballea, 1996)

Con la actual configuración de la distribución del trabajo doméstico, ello tiene un impacto particular sobre las condiciones de vida de las mujeres. Las particulares exigencias de los tiempos mercantiles interfieren con las demandas de tiempos de los trabajos domésticos generando efectos negativos para las mujeres: tensiones físicas y psíquicas en su presencia en la doble esfera, discriminación en el mercado laboral, grandes dificultades para participar en actividades extralaborales (ocio, participación socio-politica, formación, relaciones...).(Carrasco et al. 2003)

\section{Desigualdades}

Uno de los aspectos más importantes de este cambio es un aumento de las desigualdades en aspectos poco evaluados. Los cambios en la jornada laboral afectan de forma diferente a grupos sociales con diverso poder de negociación y posición social. En particular la flexibilidad temporal se impone en empleos de servicios, a menudo mal retribuidos, mientras que su extensión aumenta la disponibilidad de servicios de personas con altos ingresos. Tiene influencia sobre el reparto de la renta (puesto que se abarata el precio de la disponibilidad horaria) y la disponibilidad de tiempo libre de diversos grupos de personas. Incluso la capacidad de negociar contrapartidas está condicionada a la diferente capacidad de acción sindical imperante 
en sectores diversos. Por ello el análisis de la flexibilidad temporal debe incorporarse como un elemento significativo del análisis de las desigualdades.

\section{Reevaluar las politicas de flexibilidad}

En este artículo hemos analizado las principales líneas del debate sobre la flexibilidad laboral y los cambios que implica en materia de tiempos. Este debate sugiere algunas cuestiones básicas a las que merece dedicar atención.

En primer lugar que la defensa de la flexibilidad en general y de la flexibilidad de tiempos en particular se ha realizado considerando básicamente las ventajas que representa para empresas y consumidores, sin evaluarse adecuadamente los costes sociales que se generan a las personas en ámbitos como el desarrollo de su vida social, la organización de la vida cotidiana o la salud. Cuestiones que inciden especialmente sobre las mujeres, en la medida que no se ha producido un cambio social tan amplio que las haya liberado de su papel casi exclusivo en el cuidado de personas. Cualquier política social seria debe evaluar estos costes y tratar de eliminarlos o compensarlos.

En segundo lugar el debate ha obviado que también desde la vida cotidiana surgen necesidades de flexibilización que las empresas deberían satisfacer y que ahora simplemente se ignoran. Una política comprensiva al respecto debe aumentar el reconocimiento de los derechos de las personas a gestionar su vida cotidiana y mediar entre los derechos en conflicto.

$\mathrm{Y}$, en tercer lugar, al subrayar la demanda de adaptación al cambio, se pasa por alto que alguna de las fuentes de variabilidad lejos de ser inevitables o deseables, son males sociales que deberían atajarse (como la volatilidad extrema impuesta por los mercados financieros, o determinadas pautas de consumo socialmente indeseables o insostenibles). Se trata de proponer medidas no sólo de respuesta al cambio sino también de control del mismo, cuando tiene un carácter socialmente indeseable. En definitiva se trata de abrir el debate desde el estrecho cauce en el que se ha tratado de encerrar.

\section{BIBLIOGRAFIA:}

ABALLEA, F, (1986) "Activité de service, registre temporel et porosité des temps sòciaux" Sociologie del Lavoro 74-75 p 15-28

AKERLOF, G (1986) An Economic Theorist's book of tales Cambridge University Press

ALÓS R. de , JODAR, P (1998) "Nuevos cambios y viejas divisiones. Globalización del capital y segmentación del trabajo" en VENCE,X y OUTES, X.L.(edit.) La Uniôn europea y la crisis del estado de bienestar Síntesis, Madridi 
BETTIO,F J,RUBERY, M.SMITH (1996) "Gender, flexibility and new employment relations" Università di Siena and UMIST (fotoc)

BOSCH, G (1995) Flexibility and work organisation Social Europe Brussels

BOSCH, G y LEHNDORFF, F (1997) "La reducción de la jornada de trabajo y el empleo" Papeles de Economía Española 72, p342-365

BOYER, R. (1985) La flexibilidad del trabajo en Europa Ministerio de Trabajo y Seguridad Social, Madrid 1986

BRUNO S.(1989) La flexibilidad, un concepto contingente en M.MARUANI et al. "Debates sobre el empleo en Italia. Ministerio de Trabajo y Seguridad Social, Madrid, 1991

CARRASCO, C. et al. (2003) Trabajo, tiempo y flexibilidad: una cuestión de género Instituto de la Mujer, Col Estudios $n^{\circ} 74$, Madrid

CASTELLS, M (1999) La era de la información, $2^{a}$ edic. Alianza, Madrid

DAVIDSON, P (1994) Post Keynesian macroeconomic theory Edward Elgar, Aldershot

DOYAL L., GOUGH, I (1994) Teoria de las necesidades sociales FUHEM Icaria, Madrid

ESTEVE, f (1991) "La evolución del consumo" en M.ETXEZARRETA La reestructuración del capitalismo español 1970-1990 FUHEM Icaria, Barcelona

EUROPEAN COMISSION (1998) Flexible working and the rconcialiation of work and family life- or a new form of precariousness Employment and Social Affairs, Brussels

FINA, L (1997) El aumento del trabajo a tiempo parcial en Europa Papeles de Economia Española 72, p 67-86

GEORGE, D (2000) "Driven to spend: longer work hours as a byproduct of market forces" en GOLDEN y FIGART (2000)

GOLDEN, L. y FIGART, D.M (2000) Working time Routledge, London

HARRISON B (1997) La empresa que viene Paidós

LAVOIE, M (1992) Foundations of Post Keynesian Economics E.Elgar, Aldershot

Mc CONNELL, Campbell R. i BRUE, Stanley L (1996) Economia Laboral McGraw Hill, Madrid

MOODY, K. (1997) Workers in a lean world Verso, London

MUNOZ DEL BUSTILLO, R. et al (2003) Nuevos tiempos de actvidad y empleo Ministerio de Trabajo y Asuntos Sociales, Madrid

O.C.D.E.(1986) La flexibilidad del mercado de trabajo. Ministerio de Trabajo y Seguridad Social

O.C.D.E. (1987) Flexibilidad y mercado de trabajo. El debate actual Ministerio Trabajo y Seguridad Social, Madrid

O'REILLY, J y FAGAN, C (1998): Part-time prospects Rouledge, London.

PIORE, M. (1986) Perspectives on Labour Market Flexibility Industrial Relations,2, 
PIORE,M; SABEL, Ch. (1984) La segunda ruptura industrial Alianza, Madrid,1990

PRASCH, R.E.(2000) "Revising the labour supply curve: implications for working time and minimum wage legislation" en GOLDEN y FIGART (2000)

RECIO,A (1994) El problema de la flexibilidad laboral. Departament Economía Aplicada UAB, fotocopia

RECIO, A (2001) "Empresa red y relaciones laborales" en DUBOIS, A, MILLAN, Л y ROCA, J (edit.) Capitalismo, desigualdades y degradación ambiental Icaria, Barcelona,

SAYER,A., WALKER, R. (1992) The new social economy Basil Blackwell, Oxford

SCITOVSKY, T. (1976) Frustaciones de la riqueza Fondo Cultura Económica, MexiCo 1986

SLOANE P.J.(1978) Aspectos económicos del trabajo por turnos y de noche en países industriales Revista Internacional del Trabajo, abril

STIGLITZ, J (2002) Globalization and its discontents Penguin Press, London

THOMPSON, E.P (1979) "Tiempo, disciplina de trabajo y capitalismo industrial" en Tradición, revuelta y consciencia de clase Crítica, Barcelona 